\title{
NATO Research Task Group: 3D Scanning for Clothing Fit and Logistics
}

\author{
Allan KEEFE ${ }^{1}$, James KUANG ${ }^{2}$, Hein DAANEN ${ }^{3}$ \\ ${ }^{1}$ Defence Research and Development Canada, Toronto ON, Canada; \\ ${ }^{2}$ Department of National Defence, Ottawa ON, Canada; \\ ${ }^{3}$ Vrije University Amsterdam, Amsterdam, Netherlands
}

DOI: $10.15221 / 17.201 \quad$ http://dx.doi.org/10.15221/17.201

\begin{abstract}
Military organizations require accurate information on the relationship between body size and shape to ensure proper fit of clothing and personal equipment. From an operational perspective, proper fit is essential for soldier clothing and equipment ensembles to function as designed, allowing optimal mobility, comfort and protection from environmental and ballistic threats. Meeting these requirements is challenging, as operational uniforms and ceremonial wear must be provided to all military members. Custom tailoring is provided for individuals of extreme body size, but this practise is expensive and undesirable. Additionally, secular changes in body size, and increasing ethnic diversity and presence of women in operational trades, including combat arms, has presented a challenge to military departments responsible for clothing specification, procurement and issuing.

In response, the North Atlantic Treaty Organization Science and Technology Organization (NATO STO) has recognized the potential of 3D body scanning as a tool to rapidly acquire $3 \mathrm{D}$ anthropometry and body shape data to support clothing design and issuing. This has led to the establishment of NATO Research Task Group (RTG) HFM-266: 3D scanning for clothing fit and logistics. Currently, this Task Group is comprised of 9 member nations and one ally. The outcomes of this Task Group will serve to provide a better understating of the application of 3D body scanning technologies for military clothing and equipment application and inform the development of clothing sizing standards across NATO countries.
\end{abstract}

Keywords: 3D body scanning, NATO, standards, military logistics, clothing sizing, virtual fit.

\section{Introduction}

The logistics of specifying, procuring and issuing military clothing and personal protective equipment is a challenging task for military organizations who must ensure that adequate supplies are available to meet the needs of thousands of personnel, male and female, who work and fight in Naval, Air, Army and Special Forces environments. Of utmost importance is the assurance of proper fit across a wide range of body shapes and sizes. From a military perspective, this is important since: 1) operational clothing must provide allowances for specific occupational tasks that are posture dependent (e.g. seated postures for aircrew) without being too loose to be a snagging hazard and 2) ballistic protective equipment must be fitted over operational clothing to provide optimal coverage of vulnerable regions of the body while allowing proper mobility and integration with load carriage and additional operational-related equipment, 3) chemical and biological protective equipment has to fit properly to seal against liquid or gaseous agents and 4) ceremonial wear requires a tailored fit to meet standards of dress.

Demographic diversity within and between armed forces provides an additional challenge to military clothing logistics. Women are increasingly being integrated into army combat roles and have been established in other operational roles such as air force pilots, search and rescue technicians and clearance divers. As a result, the female body shape must be considered for clothing and equipment that was originally designed for use by male operators. An example of this is the women's version of the U.S. Army's Improved Outer Tactical Vest (IOTV) which was developed to provide a protective garment that conforms to female anatomy. Differences in body shape have also been noted between subgroups within a nation's armed forces based on ethnic [1] and linguistic [2] lines. Secular trends have demonstrated increases in body size over the past 20-30 years in Canadian [2], U.S. Army [3] and Australian [4] military personnel, creating additional challenges to maintain a current and effective clothing sizing scheme. 
3D scanning is becoming an important component of military anthropometric surveys, complementing or augmenting traditional 1D measures and providing data sets of thousands of body scans and associated demographic data. The Royal Netherlands Armed Forces (RNAF) is at the forefront of adopting 3D body scans for their clothing logistics process, as body scans are obtained from every member as part of the clothing issuing process. To date, the RNAF has amassed a database of over 12,000 body scans since 2002 [5]. Typically, one dimensional measurements are extracted from these scans to provide information for clothing issuing and specification. While advances in the development of tools to analyze 3D shape and size data are being made, the full exploitation of the shape data has yet to be realised.

In recognition of the rapid advances and acceptance of 3D body scanning technology by military organizations, the NATO Science and Technology Organization (STO) has identified 3D body scanning as a tool to rapidly acquire 3D anthropometry and body shape data to support clothing fit and logistics. This has led to the establishment of NATO Research Task Group (RTG) HFM 266: 3D scanning for clothing fit and logistics. The objectives of this Task Group are to: 1 ) identify research gaps in the area of 3D body scanning for fit and logistics, 2) facilitate integrative research and collaborative opportunities amongst member countries, 3) facilitate integrated procedures for clothing deployment to ensure similar fit over NATO countries and 4) review novel approaches for 3D scanning and processing.

\section{Clothing Logistics}

Logistics pertains to the specification, procurement, storage, and issuing of clothing of personal equipment. A good logistics system should ensure the optimal supply of clothing and equipment to fit most of the population in an effective and cost-effective manner. The cost of clothing and equipping military personnel can be substantial. For example, in the Canadian Armed Forces, dress and operational uniforms are required for over 100,000 personnel, while larger military forces such as the United States must cloth approximately 2 million personnel. Operational clothing often require specialized textiles, dyes and coatings, which drive can substantially increase the cost of each item and make second or special orders very expensive. As a result, ordering inappropriate quantity or sizes can result in significant cost to the organization.

There are many ways in which cost effectiveness can be accomplished. First, a clothing sizing system should be devised that requires as few clothing sizes as necessary while minimizing the requirement for custom tailoring for outsizes. The sizing systems should seamlessly cover the user population and sizes must be identified that are not matched to the population. Second, having a current understanding of the differences in clothing size requirements across regions or occupations is beneficial so that the proper size and quantity of clothing is available to each distribution centre. Therefore, a database of body dimension is required and an unambiguous relationship between the body dimensions and garment sizes defined. Finally, a rapid and accurate system of issuing of clothing and equipment can achieve efficiencies by reducing the number of personnel required to operate the system, reduce the amount of returns due to issuing of inappropriate clothing sizes and by providing real-time tracking of stock management and ordering.

\subsection{Clothing logistics within NATO Countries}

To gain a better understanding the current situation within NATO nations, the Task Group recently conducted a preliminary online survey of member nations to elicit current practices regarding the collection and use of body dimensions for clothing sizing and issuing. As this survey was designed to provide a snapshot of current clothing logistics practices, seven basic questions were devised, requiring a yes or no response. These questions were:

Q1. Do you use manual measurements to determine the body dimensions of military personnel?

Q2. Do your use 3D body scanning techniques to determine the body dimensions of military personnel?

Q3. Do you store the determined body dimensions of military personnel?

Q4. Do you consider your clothing and equipment sizing system to be adequate for your military personnel?

Q5. Do you notice significant complaints concerning the fit of your military clothing and/or the need for improvements in this area?

Q6. Would you appreciate a brief manual how to make and optimize a sizing system? 
To date, 36 individuals representing 20 NATO nations have responded to this survey. One null response was provided for questions 3 to 5 . In certain instances, multiple responses from a nation were received. This represents respondents with different roles within the defence organization such as scientists, project officers and clothing specialists. If at least one respondent answered "yes" to a question, then their corresponding nation was awarded an overall "yes" score. A summary of results, representing the percent "yes" scores from the 20 responding nations, is provided in Figure 1.

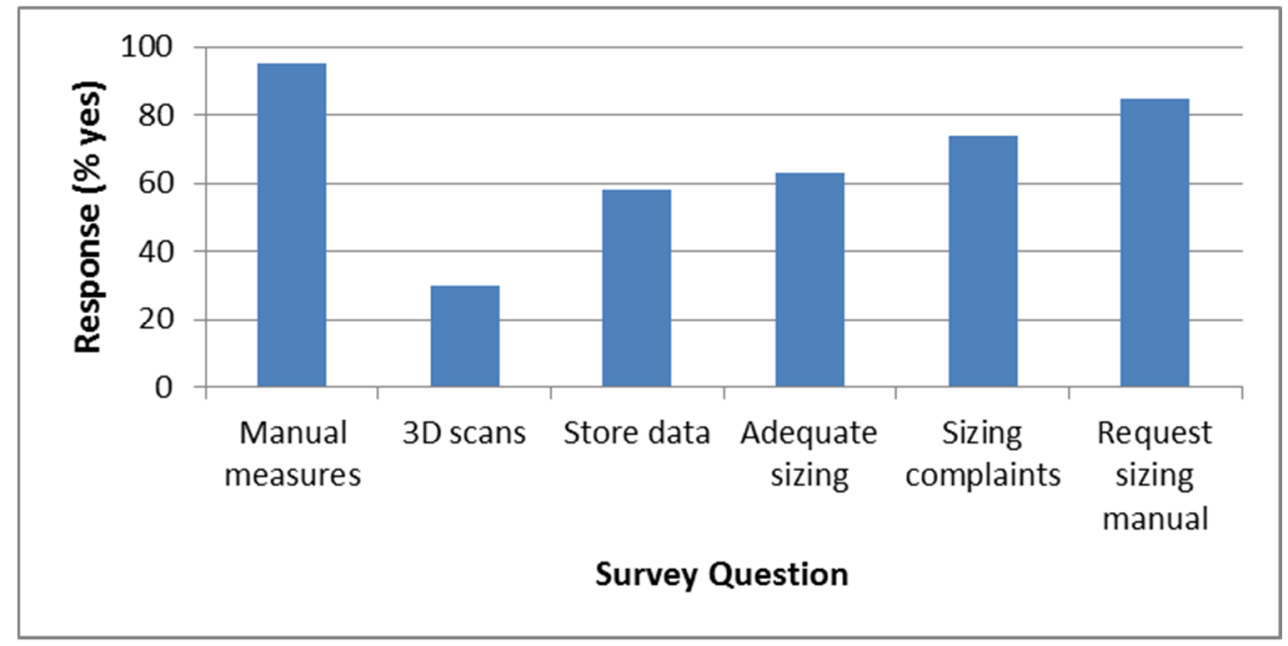

Figure 1. Summary results of the 2017 survey of 20 NATO countries on the use of body dimensions for clothing sizing and issuing.

The first three questions focused on the collection of anthropometric data. Based on these results, it is evident that almost every nation that responded (95\%) obtains body dimensions of their military personnel for clothing purposes with a little more than half $(58 \%)$ retaining these data for future use. The use of 3D body scanning is still nascent amongst respondents with $30 \%$ of nations indicate using $3 \mathrm{D}$ body scans for clothing specification and issuing. Of those nations that obtain 3D body scans, data is obtained during anthropometric surveys, scientific studies or during fitting and issuing. As mentioned previously, 3D scanning for clothing issuing is mandatory for all personnel in the Royal Netherlands Defence Force, whereas the Canada Armed Forces have body 2D photogrammetric scanning systems available in 20 locations across the country; however mandatory body scans are only implemented for fitting of specialized clothing (e.g. firefighting bunker gear).

Questions 3-6 focused on the status of the sizing systems used. Surprisingly, only 12 of the $19(63 \%)$ nations responding to this question indicate that their current sizing systems are adequate to meet their needs. While supporting comments to explain this dissatisfaction with the clothing sizing systems were not solicited, the inadequacy of these systems is reflected in the fact that $74 \%$ of respondents receive significant complaints regarding fit and sizing of clothing and equipment. Dissatisfaction amongst wearers is difficult to interpret as it could be influenced by the actual fit of garment, errors in issuing, uncomfortable physical properties of the textiles or a mismatch between users' expectation of fit and the fit as the design is intended. Importantly, common amongst most respondents (85\%) is the desire for better guidance in how to translate body dimensions into an effective and optimized sizing system.

\section{NATO Sizing Standards}

Within NATO, sizing systems are typically defined by Standardization Agreement (STANAG) documents which are used to provide a commonality of procedures and logistics across member nations and support technical interoperability. The rationale being that clothing sizing systems based on a STANAG should translate across nations. Three STANAGs of relevance to this Task Group are:

1. STANAG 2177 - Methodology for Anthropometric Data (2002).

2. STANAG 2339 - Size Marking for Interchangeability of Operational Footwear (1975), and

3. STANAG 2335 (Edition 3) - Interchangeability of Combat Clothing Sizes. 
These STANAGs are somewhat dated, referring only to sizing based on one-dimensional anthropometric measures and provide limited guidance on the relationship between fit and sizing. For example, STANAG 2335 defines fit based on the bivariate relationship between stature and chest circumference for jackets and inside leg length and waist circumference for trousers.

Gradients between sizes are specified as $5 \mathrm{~cm}$ or multiples thereof. Figure 2 provides a comparison of combat trouser sizing systems for two NATO countries.
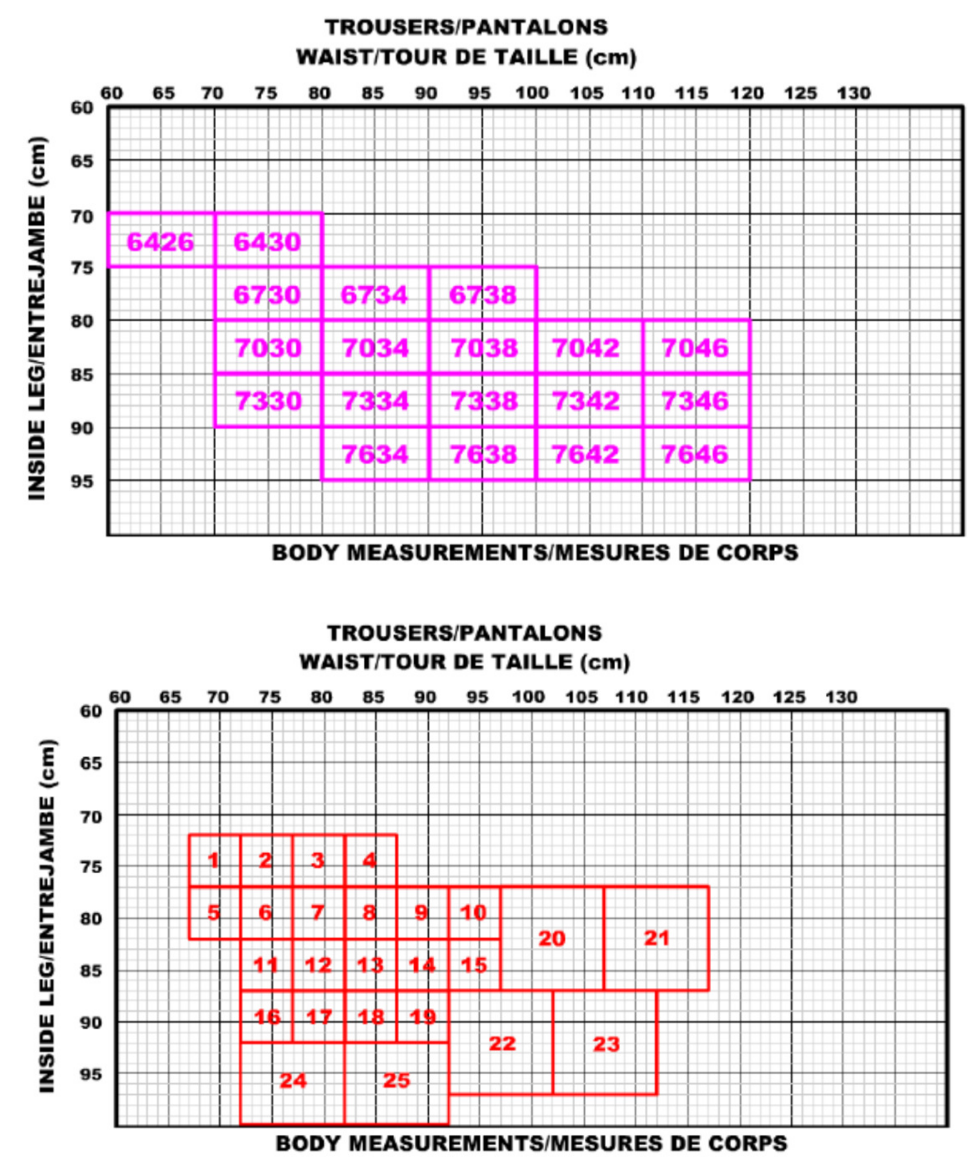

Figure 2. Combat trouser sizing schemes from two NATO countries.

As one can see, different concepts regarding in sizing systems are employed between these nations using the same NATO standard. Nation A assumes a consistent gradient step across all sizes while Nation B opts for smaller size gradients for the smaller sizes and then doubles this gradient for the larger sizes. The smaller step size of Nation B may allow for finer adjustments to fitting at the expense of requiring a greater number of sizes ( 25 sizes vs 19 for Nation A).

In addition to set body dimensions and gradient steps, STANAG 2335 is restrictive in that it only references to limited range of garments and does not provide guidance on allowance for layering of clothing ensembles.

\section{NATO Standardization Recommendation}

Previous surveys of NATO countries have revealed STANAG 2335 to be insufficient for most users and is thus rarely used. To provide updated guidance to the NATO community, the Task Group has committed to developing a document that reviews best practices and provides recommendations for the application of 3D body scanning technologies to augment the sizing and fitting of combat clothing and individual protective equipment. This document is intended as a NATO Standardization Recommendation (STANREC) and will focus on the following areas: 1) guidance on the use of multivariate data to guide design and fitting of clothing, 2) incorporation of the use of 3D data and operational assessment of fit to improve clothing fitting methods and recommend generalized methods 
for sizing methodology based on fit analysis, and 3) leveraging the guidance that is being developed in association with other international clothing sizing and 3D scanning standards through ISO TC/133 and ASTM D13 committees.

\section{Fitting and Sizing}

An effective and efficient clothing sizing system is based in determining the optimal configuration and number and size blocks that effectively accommodate the target population. This system must consider size and shape variability of the wearers, the design and material characteristics of the textiles and allowances required for layering of clothing ensemble and integration with other worn items.

\subsection{Anthropometric analysis}

Collection and proper use of anthropometric data is an important component of developing sizing systems. STANAG 2177 provides guidance on obtaining manual anthropometric measures, but the list of measures provided are limited and not necessarily sufficient for garment design or sizing. The Task Group recommends assessing these measures against current international standards such as ISO 7250 [6], and ISO 8559 [7] for completeness and use across NATO nations. It is well established that body measurements obtained by 3D scanning are not necessarily equivalent to those obtained by manual methods. Characteristics such as body hair, posture, occlusions/scan voids and movement artifacts can affect the quality of the extracted measure. Hardware and software considerations include scan resolution, template fitting, and de-noising of body scans. Automated landmarking and measurement extraction algorithms are proprietary and their accuracy and robustness across a wide range of body sizes and shapes is unknown. Research has indicated that body measurements obtained by manual techniques and scan extraction can differ depending on the measure, with greater differences being found in circumferential measures [8] as compared to linear measures [9].

ISO 20685 [10] provides standards for maximum allowable error between extracted and manually measured values, however, in practice these limits are difficult to achieve for the reasons mentioned above. The anthropometric data used for the development of sizing systems are typically based on manual measures obtained from national surveys, the effect of differences between measurement technologies on clothing sizing and issuing must be examined. The Task Group recognizes that a better understanding of the differences between manual and 3D body measures is important in order to define and compare clothing sizing systems derived from data using either of these two methods.

As mentioned previously, STANAG 2335 recommends sizing systems based on the bivariate relationship between standardized anthropometric variables. This is limiting in that it this neglects the multivariate aspect of clothing fit. In the example of a combat shirt, waist circumference may be confounding factor resulting in individuals with a larger belly selecting a larger size shirt to accommodate their girth. To account for this, various multivariate approaches to develop sizing systems such as multiple regression, cluster analysis and principal components analysis have been proposed. For a comprehensive overview of these and other clothing sizing techniques, see Zakaria and Gupta [11]. An assessment of these methods, with the possible incorporation of 3D body shape analysis techniques, needs to be performed to identify best practises and areas for further research.

Extracting body measures from 3D scans provides a rapid and reliable method to obtain anthropometric data, however, its limitation lies in the fact that valuable body shape information is often discarded, especially when analyzing large population-based datasets. The visualization and manipulation of aggregated 3D shape data has been limited to research institutes who have developed analytical tools based on Principal Components Analysis (PCA) techniques pioneered by Allen [12]. Recently, the Canada [13] and the United States [14] military have developed PCA-based tools which will be available to their procurement specialists to inform clothing and equipment specification. Each of these tools allow the user to explore the size and shape variability within a 3D scan database and extract relevant body dimensions from the specified shape model (Figure 3). 

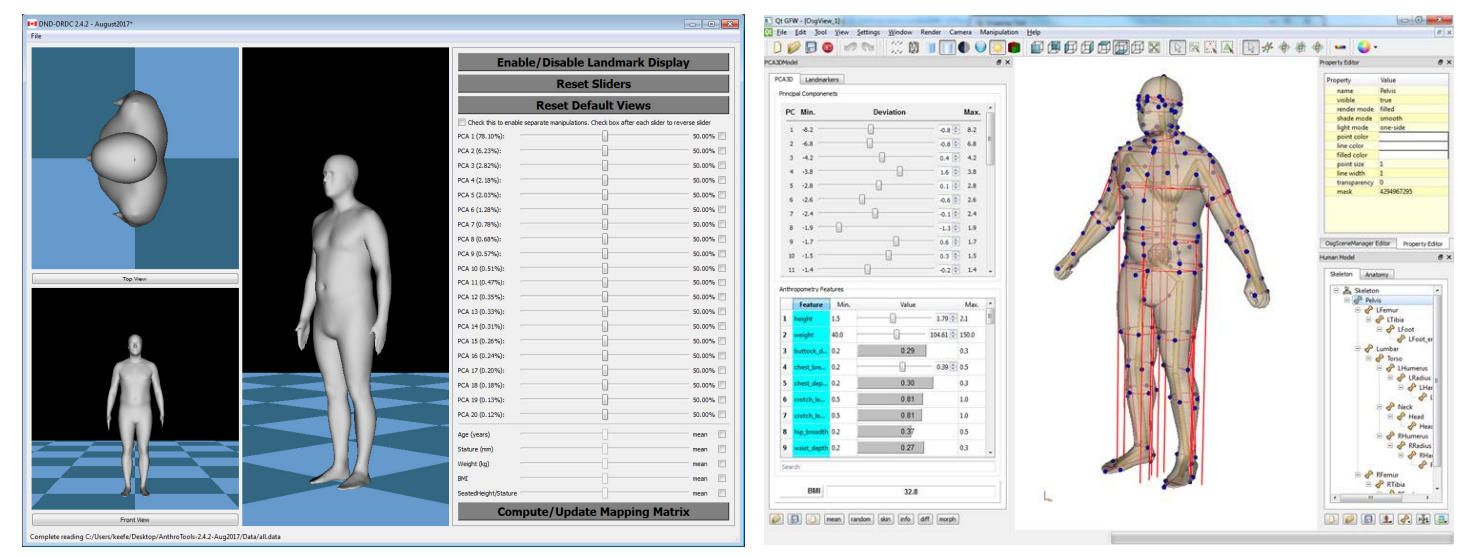

Figure 3. PCA-based tools developed by Canada (left) and the United States (right) military to support acquisition.

As the use of PCA-based shape tools for use in clothing and equipment design is in its infancy, it is anticipated that further research and development of design guidelines are required to ensure that these tools may be fully exploited.

\subsection{Sizing systems}

Referring back to the NATO survey illustrated in Figure 1, it is apparent that there is a general dissatisfaction with sizing systems across member nations. The reasons for this are varied. Secular changes in the user population, personal preference of fit, poor consideration of female body shape, changes in textiles and protection factors may all be culprits. Of course, one must consider that the original sizing scheme may have been poorly conceived in the first place. The challenge of developing sizing schemes that are satisfactory to a wide range of the population is well known in the commercial field. Recently, BODI.ME [15] published a survey on clothing returns in e-fashion. One of many interesting findings is that $42 \%$ of consumers that purchase clothing on-line return items due to poor fit. Whether this is due to insufficient sizing schemes, or lack of consumer education $(40 \%$ of online shoppers did not consult sizing charts), is open for debate. A military analogy to this is the clothing stores clerk who issues clothing based on visually sizing up a recruit and making a size determination based on experience rather than objective data. Another factor that results in sizing/selection mismatch is the wearer's subjective preference of fit or appearance. In some cases, a soldier may opt for a smaller size to give a form-fitting appearance, whereas another soldier may select a larger size to allow for more comfort when working in extreme postures. In both cases, the selection of inappropriate size clothing may compromise the intended performance of the garment. Too tight or small may restrict motion or provide insufficient thermal or ballistic protection, while too loose may constitute a snagging hazard. Thus, it is important that proper education on the how's and why's of proper fit is important to ensure that users follow the proper fitting guidelines.

The Task Group maintains that clothing should be sized based on the interaction between the user and the garment. This can be accomplished by conducting a fit mapping process to quantify optimal garment size and design and identify optimal size schemes and tarrifs. Fit mapping is "the iterative process of applying fit testing to improve the fit quality of a garment and classify who fits and does not fit in test sizes" [16]. The determination of a size scheme is based on empirical factors such as: 1) an objective definition of the concept of fit in terms of lines and ease, 2) consideration of all aspects of body size and shape, 3) performing occupationally relevant static and dynamic evaluations of fit, 4) analysis of the results and 5) classification of body types to sizes and review of the garment design to allow improvement to fit.

Virtual fitting technology is of great interest to the Task Group as it can contribute to the fit mapping process by providing additional insight into the relationship between body shape, garment design, sizing and fit. Daanen et al. [17] conducted an investigation into the relationship between the predicted fit of a combat shirt and trouser with subjects preferred size. Their findings revealed that the shirt fit determined by body scan dimensions did not map particularly well to the subjects preferred clothing size, whereas, there was better agreement with the combat pants. As a result, a recommendation was made to adjust the pattern for the shirt to allow for better agreement between fit and size. This was followed by a virtual fitting exercise using the Modaris 3D fit software (Lectra, 
Paris, France) to assess the ease of the clothing preferred by the subjects. Figure 4 provides a comparison of proper fitting pants to the fit of a subject who selected pants with size too small of a waist size. Thus, the incorporation of 3D body scanning and a virtual fit procedure provided an objective methodology to identify clothing fitment issues.

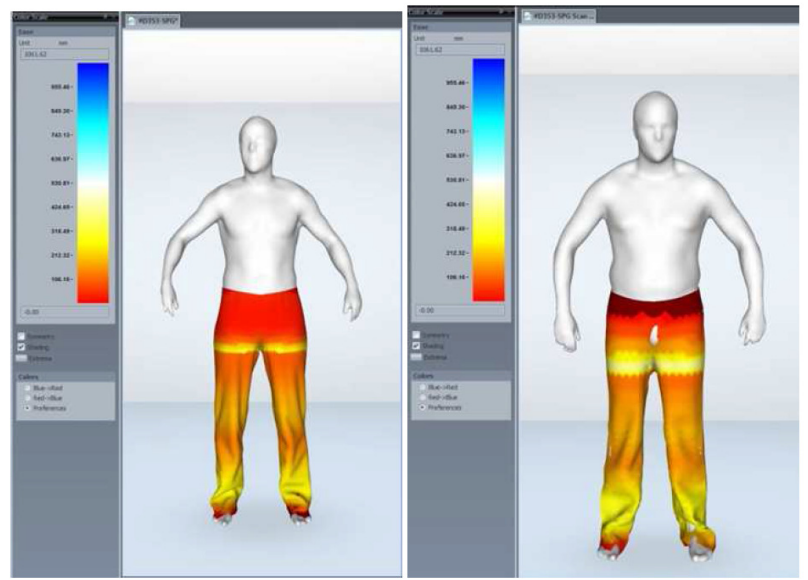

Figure 4. Virtual fit example of properly (left) and inappropriately (right) trousers [17]. Reprinted with permission.

\subsection{Technology considerations}

One of the key questions of interest to the Task Group is which body scanning technology is the most suitable for use in the military clothing logistics chain. The pace of technological development has increased so rapidly that a review of 3D scanning systems by Daanen and Terhaar [18] published only four years ago is under consideration for update. Low cost photogrammetric systems and depth sensing technologies have pushed the cost of acquiring 3D imaging within the range of the most budget conscious. 3D body scanning capability is now available as a smart phone app, where consumers can determine body dimensions or create custom avatars based on simple photographs. In fact, technology has advanced such that accurate 3D avatars can be created, without the need for body scanning. An accurate 3D model can be generated by providing a few simple manually obtained body dimensions [19], user provided height and weight [20] or extracted from a single image [21].

Of interest to the Task Group is the value of obtaining 3D body shape data in operationally related poses in addition to the standard " $A$ " pose required by many body scanning systems. One technology of interest is 4D dynamic body scanning technology which can obtain dynamic 3D scans of the body throughout a task range of motion. Conversely, prediction of body motion and tissue deformation can also be achieved through predictive algorithms [22]. This is particularly important for the development of operational clothing, protective equipment, exoskeletons, foot, head, and hand wear where fit and performance must be maintained across the wearer's full range of motion.

Concurrent with hardware advancements, the Task Group is actively monitoring advances in 3D scan processing and data management software. There are many commercial and open source tools available to visualize and process $3 \mathrm{D}$ body scans, while commercial software to extract body dimensions, and perform 3D shape analysis, virtual design and virtual fitting are constantly evolving.

The question of which technology or system is most suitable for a military clothing logistics system has yet to be determined, however, it is quite likely that a combination of solutions may be necessary. For body scans that are to be incorporated into a database for research or detailed shape analysis purposes, a high-resolution system with sophisticated post processing software may be required. Clothing supply depots or recruitment centres which offer a kiosk service for clothing issuing may be best served by a simpler, cost effective solution. This is especially important for countries which are geographically dispersed and require the installation of a network of scanning systems. Finally, a simple smartphone or manual measurement, web-based system may be sufficient for servicing military members in remote communities who do not have convenient access to a clothing supply depot and need to order garments online. 


\subsection{Scanner requirements}

Incorporating a 3D body scanning system into a military logistics system can be a complex undertaking. While countries with a smaller geographical area may suffice with a centralized issuing and distribution facility, the cost and complexity of managing a system of 3D scanning systems will increase substantially if a distributed network of body scanning facilities is required.

Factors such as initial purchase cost, care and maintenance and lifecycle management must be taken into consideration. From a personnel perspective, knowledgeable operators must be trained to facilitate the operation of the scanning system and provide a quality assurance/control function. Care must be taken to ensure that a participant's modesty and privacy is maintained during the scan procedure as well as protecting their data from unauthorized access. Finally, if the scan data is to be stored and used for assessing or specify future clothing and equipment acquisition, a comprehensive data management and analysis plan must be in place to ensure that the data is of sufficient quality and quantity for statistical modeling purposes.

The following is a preliminary list of requirements identified by the Task Group for 3D body scanning systems incorporated into a clothing logistics system.

For military use, the following shall comply,

1. Automatically identify common body landmarks and provide anthropometric data collection functionality.

2. Provide a feature that users can import custom clothing size charts so that an individuals predicted clothing sizes can be automatically generated.

3. Robust hardware and software that is easy to install, operate, and maintain.

4. Allows for a private and comfortable scan experience.

5. Provision of security for an individual's data.

6. Accurate and automatic post-scan data processing.

7. Safety: eye and skin safe.

8. Data output format interchangeable with other software programs, allows freedom to choose third party software if required.

9. Stability toward working environment (e.g. ambient light condition, temperature change etc.)

10. Warranty and onsite maintenance and support.

The following should comply,

1. High speed scan acquisition to avoid movement effects.

2. Measuring sitting posture capability.

3. Automated calibration procedure, if calibration required.

5. Smallest footprint as possible (to minimize space use).

6. Self-serve kiosk capability.

6. Database and statistics capability to review and analyse stored data.

8. Networked for backup and maintenance of multiple, distributed systems.

While this list is far from complete, it incorporates key requirements for military organizations to consider for future 3D body scanning acquisition and operation. As the Task Group continues to review 3D body scanning technologies and concepts for visualizing and analyzing the resulting body size and shape data, it is anticipated that this list will be refined to meet the needs of small and large military organizations and the research and operations communities within them.

\section{Conclusion}

NATO HFM RTG 266 is an international Task Group focusing on the application of 3D scanning to support military clothing fit and logistics of NATO countries and their allies. To date, the Task Group has conducted a survey on the use of anthropometric data for clothing sizing and issuing within NATO countries and has identified a requirement for the modernization of NATO Standardization Agreements pertaining to clothing sizing and anthropometry. An objective of the Task Group is to develop a NATO Standardization Recommendation (STANREC) document to provide critical advice to member nations on the use of 3D body scanning technology and the development of clothing sizing schemes. It is anticipated that this will result in improved fit and performance of military clothing and achieve efficiencies in the acquisition and issuing of these items. 


\section{References}

[1] C.C. Gordon, T. Churchill, C.E. Clauser, B, Bradtmiller, J.T. McConville, I. Tebbetts, and R.A. Walker. 1988 Anthropometric survey of U.S. Army personnel: methods and summary statistics. NATICK/TR-89/044. Natick, MA: U.S. Army Natick RD\&E Center. 1989.

[2] A.A. Keefe, H. Angel, B. Mangan. 2012 Canadian Forces anthropometric survey (CFAS): final report. DRDC-RDDC-2015-R186. Defence Research and Development Canada. 2015.

[3] C.C. Gordon, C.L. Blackwell, S.P. Paquette, J.L. Parham, and B. Bradtmiller. "Anthropometric change in the US Army 1988-2012: implications for design". In Proc. 2015 19th Triennial Congress of the International Ergonomics Association. Melbourne, 9-14 August.

[4] G. Tompkinson, A. Clark, and P. Blanchonette. Body size changes of Royal Australian Air Force aircrew: 1971-2005. DSTO-RT-2339. Defence Science and Technology Organization. Fishermans Bend, Victoria 3207 Australia. 2009.

[5] F.B. Ter Haar, H.G.B Reulink, and H.A.M Daanen. 3D scanning of dutch military - secular trends in PCA for 18,000 soldiers. In Proc 2013 4th International Conference on 3D Body Scanning Technologies, Long Beach CA, USA, 19-20 November.

[6] ISO. International Standard ISO 7250-1:2008(E). Basic human body measurements for technological design - Part 1: Body measurement definitions and landmarks. International Organization for Standardization. Geneva, Switzerland. 2008.

[7] ISO. International Standard ISO 8559:1989(E). Garment construction and anthropometric surveys- Body dimensions. International Organization for Standardization. Geneva, Switzerland. 1989.

[8] H. Han, Y. Nam and K. Choi. Comparative analysis of 3D body scan measurements and manual measurements of size Korea adult females. Int. J. of Industrial Ergonomics, 40, 530-540. 2010.

[9] K. Robinette and H. Daanen, Precision of the CAESAR scan-extracted measurements, Applied Ergonomics, 37(3):259-265, 2006.

[10] ISO. International Standard ISO 20685:2010(E). 3D scanning methodologies for internationally compatible anthropometric databases. International Organization for Standardization. Geneva, Switzerland. 2010.

[11]N. Zakaria and D. Gupta. "Apparel sizing: existing sizing systems and the development of new sizing systems" in Anthropometry, apparel sizing and design. Cambridge, UK: Woodhead Publishing Ltd., 2014, pp. 3-33.

[12]B. Allen, B. Curless, and Z. Popovic, The space of human body shapes: reconstruction and parameterization from range scans, ACM Transactions on Graphics, 22(3): 587-594, 2003.

[13] C. Shu, P. Xi, and A. Keefe. Data processing and analysis for the 2012 Canadian Forces 3D anthropometric survey, International Conference on Applied Human Factors and Ergonomics (AHFE 2015), Procedia Manufacturing, 3:3745-3752. 2015.

[14]X. Zhou, K. Sun, P.E. Roos, P. Li, and B. Corner. Anthropometry model generation based on ANSUR II database. Int. J. digital Human, Vol. 1, No. 4, pp. 321-343. 2017.

[15]BODI.ME, London, U.K. "Survey Report: The dilemma of online shopping, ". 2017 [Online]. Available: http://fashion.bodi.me/wp-content/uploads/2017/03/Bodi.Me-Survey-Report-Jan2017.pdf.

[16] H.J. Choi, J.A Hudson, and G.F. Zehner. A manual for the performance of protective equipment fit-mapping. AFRL-RH-WP-SR-2010-005. Air Force Research Laboratory. 2009.

[17]H.A.M. Daanen, A. Woering, F.B. Ter Haar, A.A.M. Kuijpers, J.F. Haker, and H.G.B. Reulink. Optimization of military garment fit. Ambience`14 \& 10i3m, 7-9 Sept Tampere, Finland. 2014.

[18] H.A.M. Daanen, and F.B. Ter Haar. 3D whole body scanners revisited. Displays 34. 270-275. 2013.

[19]S. Wuhrer and C. Shu. Estimating 3D human shapes from measurements. Machine Vision and Applications, 24(6):1133-1147, 2013.

[20]Body Labs, New York. "Body Labs blue one sheet," 2016. [Online]. Available: https://www.bodylabs.com/wp-content/uploads/2016/09/Body-Labs-Blue-Data-Sheet.pdf, Accessed on: Aug 23, 2107.

[21]P. Guan, A Weiss, A. Balan, and M. Black, "Estimating human shape and pose from a single image", Int. Conf. on Computer Vision, ICCV, Kyoto, Japan, Sept. 2009.

[22] M. Loper, N. Mahmood, J. Romero, G. Pons-Moll, M. Black. SMPL: A Skinned Multi-Person Linear Model. ACM Trans. Graph. 34, 6, Article 248, 16 pages, 2015. 